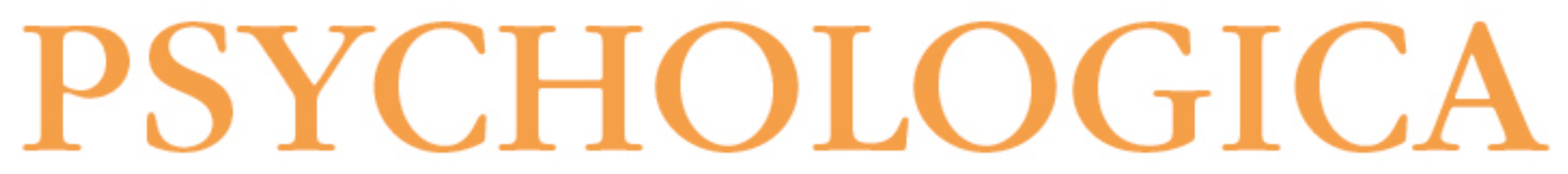

\title{
Transição e adaptação ao ensino superior e a demanda pelo sucesso nas instituições portuguesas
}

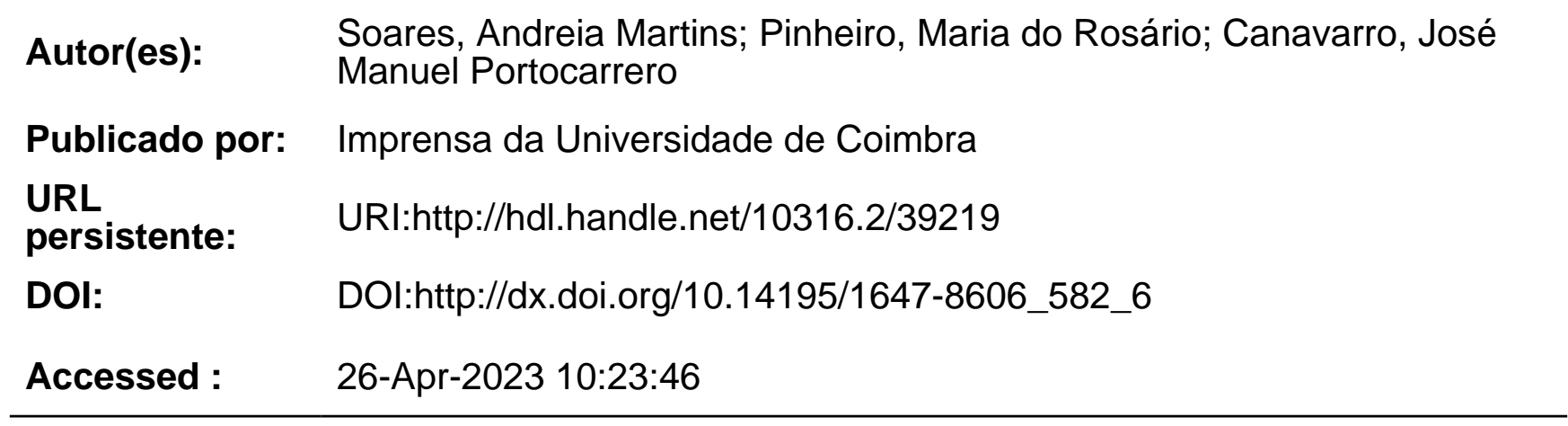

A navegação consulta e descarregamento dos títulos inseridos nas Bibliotecas Digitais UC Digitalis, UC Pombalina e UC Impactum, pressupõem a aceitação plena e sem reservas dos Termos e Condições de Uso destas Bibliotecas Digitais, disponíveis em https://digitalis.uc.pt/pt-pt/termos.

Conforme exposto nos referidos Termos e Condições de Uso, o descarregamento de títulos de acesso restrito requer uma licença válida de autorização devendo o utilizador aceder ao(s) documento(s) a partir de um endereço de IP da instituição detentora da supramencionada licença.

Ao utilizador é apenas permitido o descarregamento para uso pessoal, pelo que o emprego do(s) título(s) descarregado(s) para outro fim, designadamente comercial, carece de autorização do respetivo autor ou editor da obra.

Na medida em que todas as obras da UC Digitalis se encontram protegidas pelo Código do Direito de Autor e Direitos Conexos e demais legislação aplicável, toda a cópia, parcial ou total, deste documento, nos casos em que é legalmente admitida, deverá conter ou fazer-se acompanhar por este aviso.

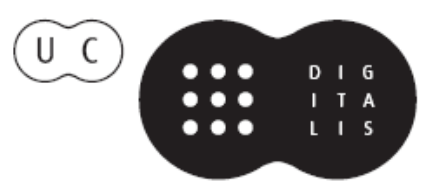


VOLUME $5 \bigcirc$ No2 2015

\section{PSYCHOLOGICA}

IMPRENSA DA UNIVERSIDADE DE COIMBRA

COIMBRA UNIVERSITY PRESS

FACULDADE DE PSICOLOGIA E DE CIÊNCIAS

DA EDUCAÇÃO DA UNIVERSIDADE DE COIMBRA 


\title{
Transição e adaptação ao ensino superior e a demanda pelo sucesso nas instituições portuguesas
}

\author{
Andreia Martins Soares ${ }^{1}$ Maria do Rosário Pinheiro² e José Manuel \\ Portocarrero Canavarro ${ }^{3}$
}

Transition and adaptation to higher education and the quest for success in Portuguese institutions

\begin{abstract}
Despite the many important changes occurred in Portuguese higher education institutions since the last years of Estado Novo (New State) and the efforts to accomplish the demanding mission of preparing students for the challenges of the Europe of Knowledge, several indicators put the Portuguese higher education far from an educational system that is intended to be inclusive, differentiated and of high quality. The national research focused (1) on the understanding of academic success and of transition and adjustment to the higher education process and (2) on subsequent planning / implementation of actions to promote them has been a good contribution to the prosecution of previously mentioned pretensions. This review article will address these complex phenomena, emphasizing the latest research perspectives and results, as well as the answers that national postsecondary institutions have developed to promote academic success (which, in its broader meaning, includes a successful transition and adaptation process).
\end{abstract}

Keywords: adaptation; higher education; institutional support structures; academic success; transition

1 Faculdade de Psicologia e Ciências da Educação da Universidade de Coimbra, Portugal, Bolseira de Doutoramento (FCT - SFRH/BD/47228/2008). Email: andreiamsoares2@gmail.com

2 Faculdade de psicologia e Ciências da Educação da Universidade de Coimbra, Portugal. Email: mrpinheiro@fpce.uc.pt

3 Faculdade de Psicologia e Ciências da Educação da Universidade de Coimbra, Portugal. Email: josecanavarro@fpce.uc.pt 


\section{Resumo:}

Não obstante as importantes transformações operadas nas instituições portuguesas de ensino terciário, desde os últimos anos do Estado Novo, e os esforços para cumprir a exigente missão de preparação dos seus estudantes para os desafios da Europa do Conhecimento, diversos indicadores colocam o ensino superior nacional por agora longe de um sistema de ensino que se pretende inclusivo, diferenciado e de grande qualidade. A investigação nacional sobre o sucesso académico e o processo de transição e adaptação ao ensino superior, centrada na compreensão destes fenómenos e subsequente planeamento/aplicação de intervenções neste âmbito, tem sido um bom auxílio à persecução das pretensões anteriores. Neste artigo de revisão abordar-se-ão estes complexos fenómenos, enfatizando as perspetivas adotadas e os resultados alcançados na investigação mais recente, bem como as respostas que as instituições nacionais de ensino terciário têm desenvolvido no sentido de promover o sucesso académico que, na sua aceção mais ampla, inclui um processo de transição e adaptação bem-sucedido.

Palavras-chave: adaptação; ensino superior; estruturas de apoio ao aluno; sucesso académico; transição

\section{INTRODUÇÃO}

O ensino superior, na Europa e em Portugal, tem sofrido inúmeras transformações desde a criação das primeiras instituições de ensino superior. Em Portugal, ainda durante os últimos anos do Estado Novo, iniciou-se o processo de expansão (institucional, decorrente do lançamento de novas instituições de ensino superior, e territorial, através do aumento destas instituições no interior do país), diversificação (no que concerne à formação disponível para novas áreas) e complexificação (do modelo de organização) deste segmento de ensino (Amante, 2007; Rego \& Caleiro, 2010). Mais recentemente, as instituições de ensino superior portuguesas (e congéneres europeias) têm procurado integrar (reformulando e reorganizando objetivos, finalidades e atitudes/práticas) as alterações de políticas de ensino e estruturais, impulsionadas pelos compromissos selados na Declaração de Sorbonne e na Declaração de Bolonha (datadas de 1998 e 1999, respetivamente) (Amante, 2007), num esforço conjunto de construção de um espaço europeu coerente e compatível de ensino superior ${ }^{4}$. Assim, se até ao século XIX a missão das instituições

4 Este espaço europeu de ensino superior é amplamente valorizado por ser entendido como um meio auxiliar na promoção da empregabilidade e da mobilidade na Europa, assim como da competitividade internacional e da atração de estudantes do mundo (Ponte, 2005). 
de ensino terciário se centrava exclusivamente na transmissão de conhecimentos, atualmente, e de acordo com a legislação portuguesa em vigor ${ }^{5}$, têm por objetivo a qualificação de alto nível dos seus estudantes, a produção e difusão do conhecimento e a formação cultural, artística, tecnológica e científica dos seus discentes, num quadro de referência internacional, devendo ainda valorizar a atividade dos que nelas trabalham e estudam, assegurar o acesso igualitário à aprendizagem ao longo da vida, promover a mobilidade nacional e internacional de estudantes e diplomados, participar em atividades de ligação à sociedade e contribuir para a compreensão pública das humanidades, das artes, da ciência e da tecnologia.

Perante o seu importante papel de coprotagonista na construção de uma Europa do Conhecimento (Comissão das Comunidades Europeias, 2003) e a sua exigente missão, conhecer o êxito das instituições do ensino superior, na persecução desses intuitos, revela-se uma tarefa necessária e torna o ensino superior num objeto de variadas análises (Lobo, Almeida, \& Pinheiro, 2011). O confronto periódico com os indicadores de input e output (educativos, formativos, científicos, tecnológicos, financeiros, económicos e sociais) que, quase invariavelmente, colocam Portugal nos últimos lugares, a par com alguns países do leste da Europa, das tabelas dos diversos países em comparação ${ }^{6}$, parece sugerir que ainda há um longo caminho a percorrer pelas jovens instituições portuguesas de ensino superior, na persecução de um sistema inclusivo, diferenciado e de grande qualidade (Crespo, 2006). Não obstante, muitos têm sido os esforços para atenuar esta distância. Entre esses esforços, encontra-se o significativo investimento de recursos na investigação de (e na intervenção sobre) dois fenómenos complexos: o sucesso académico e o processo de transição e adaptação ao ensino superior.

Neste artigo de revisão discutir-se-ão os fenómenos anteriores, enfatizando as perspetivas adotadas e os resultados alcançados na investigação mais recente, bem como as respostas que as instituições nacionais de ensino terciário têm desenvolvido no sentido de promover o sucesso académico que, na sua aceção mais ampla, inclui um processo de transição e adaptação bem-sucedido.

\section{SUCESSO ACADÉMICO NO ENSINO SUPERIOR: CONCEITO AMPLO E FENÓMENO DE CAUSAS MÚLTIPLAS E COMPLEXAS}

A definição de sucesso (bem como de insucesso) académico permanece um desafio já que existem várias perspetivas sobre o mesmo (Brites, Seco, Canastra,

5 Conferir Artigo $2^{\circ}$ da Lei no 62/2007, expressa no Diário da República n 174 Série I de 10/09/2007.

6 A título de exemplo, sugere-se a leitura do relatório da Organização de Cooperação e de Desenvolvimento Económico (adiante, OCDE) - Regards sur léducation 2015: Les indicateurs de l'OCDE. 
Dias, \& Abreu, 2010), que originam diferentes operacionalizações do conceito e consequentemente dificultam a comparação de alguns estudos sobre este tema.

Parece existir um consenso alargado sobre a necessidade de uma definição mais ampla desta representação mental, que não se confunda com outros conceitos mais restritos como o rendimento/desempenho/sucesso escolar, entendido como uma das dimensões do sucesso académico e aquela que envolve essencialmente as classificações obtidas pelos alunos nas diferentes disciplinas (Brites et al., 2010; Silva \& Ribeiro, 2007; Tavares \& Huet, 2001). Para a maioria dos autores, o sucesso é de natureza multifacetada, devendo a sua apreciação incluir aspetos escolares, sociorrelacionais e psicológicos (Almeida, 2002), como os que seguidamente se particularizam: (1) o desenvolvimento de competências intelectuais (pensamento crítico, resolução de problemas, tomada de decisão refletida, entre outras) e académicas, (2) o estabelecimento e manutenção de relacionamentos interpessoais positivos e gratificantes, (3) o desenvolvimento da identidade e (4) da autonomia em direção à interdependência, (5) a decisão por um determinado projeto de carreira/profissional e a perspetiva de um estilo de vida próprio, (6) o usufruto de saúde e bem-estar, (7) o desenvolvimento de uma filosofia integrada de vida e (8) o progresso no próprio processo de transição e adaptação ao ensino superior (Ferreira, Almeida, \& Soares, 2001; Pinheiro, 2003; Upcraft, Gardner, \& Barefoot, 2005).

No que concerne às causas explicativas/fatores preditores e associados a este fenómeno, os resultados de diversos estudos mostram alguma coerência, apontando para uma constelação de fatores (cf., e.g., Araújo \& Almeida, 2010; Bruinsma \& Jansen, 2007; DeBerard, Spielmans, \& Julka, 2004; Luo, Williams, \& Vieweg, 2007; Pacheco, 2008; Pinheiro, 2003; Ruthig, Marrone, Hladkyj, \& Robinson-Epp, 2011; Silva \& Ribeiro, 2007; Soares, Almeida, Diniz, \& Guisande, 2006; Veloso, Costa, \& Lopes, 2010), ainda que a divisão, integração e organização das variáveis possa diferir, consoante a perspetiva utilizada para a leitura do fenómeno (Brites et al., 2010). A maioria destas perspetivas é influenciada pelas teorias ecológicas, nomeadamente pelo Modelo Ecológico de Bronfenbrenner ${ }^{7}$ (citado em Canavarro, 2007), no que se refere à interação entre o indivíduo e o ambiente.

Assim, no quadro conceptual integrador que propõe, Alarcão (2000) sistematiza os fatores (considerados interdependentes e influenciáveis pelas ideologias, valores e preconceitos vigentes) associados ao sucesso académico, em função de quatro dimensões principais: alunos (e.g., condições familiares e socioeconómicas, integração social, autonomia na gestão do estudo, adequação dos métodos de estudo, participação em atividades extracurriculares, articulação entre valores, estilo de

7 Segundo o Modelo Bio-Ecológico de Bronfenbrenner, há quatro componentes fundamentais que devem ser consideradas no desenvolvimento humano: os processos (formas particulares de interação entre organismo e ambiente), a pessoa (com as suas disposições, recursos e exigências), o tempo e os contextos (do micro ao macrossistema) (Canavarro, 2007). 
vida, etc.), docentes (e.g., relação com os alunos, competência científica e pedagógica, adequação entre ensino e avaliação, as expectativas em relação aos alunos, etc.), currículo (e.g., articulação entre níveis de ensino, existência de pré-requisitos adequados, organização de calendários e horários, flexibilidade curricular, etc.) e instituição universitária (e.g., condições físicas, dimensão das turmas, instrumentos de trabalho, coordenação das diversas estruturas, etc.). No mesmo sentido, Tavares e Silva (2001) adotam uma visão micro-meso-exo-macrossistémica ecológica e dialógica e apontam também os alunos, os docentes, o currículo, as instituições, as políticas educativas e as ideologias como causas ou fatores de sucesso.

Já Soares, Almeida, Diniz e Guisande (2006) mencionam outras categorias de fatores: os inerentes aos estudantes (características sociodemográficas, académicas e desenvolvimentais), as características de qualidade da instituição (infraestruturas, serviços, recursos), a interação entre estes dois grupos de características e o envolvimento dos estudantes (i.e., ao grau de investimento cognitivo e comportamental que os estudantes esperam dedicar ou dedicaram à vida universitária).

Por sua vez, Taveira (2000) categoriza as variáveis associadas ao fenómeno do sucesso académico em dois grandes grupos: individuais (fatores biodemográficos e académicos, personalidade e papéis) e contextuais (contextos universitário, familiar e de pares). De forma semelhante, Brites et al. (2010), influenciados pelas categorizações anteriores, apresentam duas classes de variáveis: as relacionadas com a instituição (equipamentos e serviços, estruturas de apoio ao aluno, organização curricular, práticas pedagógicas, preparação científica e pedagógica dos docentes, atividades extracurriculares, etc.) e as relacionadas com o indivíduo (transição para o ensino superior, fatores contextuais e individuais).

Tomando por sensata qualquer uma das leituras/categorizações anteriores e recorrendo às detalhadas revisões de estudos sobre o que afeta o sucesso académico e a permanência do estudante no ensino superior (e.g., Astin, 1997; Brites et al., 2010; Curado \& Machado, 2005; Ishler \& Upcraft, 2005; Pascarella \& Terenzini, 1991, 2005), bem como aos resultados de estudos mais recentes de associação e predição estatística que incidem sobre a temática (e.g., Bruinsma \& Jansen, 2007; DeBerard et al., 2004; Gold \& Souvignier, 2005; Mills, Heyworth, Rosenwax, Carre, \& Rosenberg, 2009; Nunes, 2006; Soares, Almeida, Diniz et al., 2006) ou do estudo de casos sobre fatores organizacionais, representações dos atores e práticas de promoção do sucesso escolar (cf. Veloso et al., 2010), é possível detalhar, de forma sucinta, algumas das variáveis que sistematicamente surgem associadas ou são preditoras do sucesso académico.

Por conseguinte, dentro do grupo das variáveis relacionadas com o indivíduo, os resultados dos estudos apontam, entre outros: (1) o sucesso escolar anterior (medido a partir do desempenho obtido no ensino secundário, da nota de candidatura ao ensino superior ou das primeiras avaliações no ensino superior) como 
forte preditor do sucesso escolar (e.g., Bruinsma \& Jansen, 2007; DeBerard et al., 2004; Gold \& Souvignier, 2005; Pascarella \& Terenzini, 1991, 2005), (2) determinadas características biosociodemográficas (e.g., ser um estudante tradicional mais novo e/ou do género feminino, proceder de uma família com uma situação social e económica e um património cultural e académico mais favoráveis) como facilitadoras do acesso e da passagem próspera pelo ensino superior (e.g., Almeida, Guisande, Soares, \& Saavedra, 2006; Astin, 1997; Mills et al., 2009; Veloso et al., 2010), (3) o importante papel da perceção do suporte social e do encorajamento da família e dos amigos e de outros significativos nesse sucesso (e.g., DeBerard et al., 2004; Figueiredo, Maia, \& Pinheiro, 2004, 2011; Pinheiro, 2003, 2004; Pinheiro \& Ferreira, 2002, 2006; Rayle \& Chung, 2008; Seco, Casimiro, Pereira, Dias, \& Custódio, 2005). Alguns estudos sublinham ainda (4) a importância da motivação, da perceção de autoeficácia, das competências autorreguladoras da aprendizagem e da abordagem ao estudo no sucesso académico, sugerindo que os alunos mais motivados (particularmente os que concretizaram os seus desejos e expetativas no que concerne à prioridade da escolha do curso/instituição de ensino), com perceções positivas de autoeficácia, autorreguladores da sua aprendizagem e que adotam uma abordagem profunda ao estudo têm maiores probabilidades de serem bem-sucedidos (e.g., Bruinsma \& Jansen, 2007; Pinheiro, 2010; Santos \& Pinheiro, 2010; Rosário, Nunes, et al., 2010; Rosário, Núñez, et al., 2010; Seco et al., 2005; Veloso et al., 2010; Zajacova, Lynche, \& Espenshade, 2005; Zimmerman, 2008).

Quanto ao grupo das variáveis contextuais, Pascarella e Terenzini (1991, 2005) concluíram que certas variáveis institucionais (e.g., seletividade, duração da oferta formativa, tamanho e controlo público vs. privado, composição racial/género) conduzem melhor o estudante para a sua permanência no ensino superior do que outras (e.g., instituições com critérios de seleção mais rigorosos, com oferta formativa de maior duração, com composições mais homogéneas tendem a diminuir a probabilidade de insucesso e de abandono), ainda que o peso da influência destas variáveis seja inferior ao das experiências do estudante depois do ingresso. Também a existência de serviços ou unidades orgânicas de apoio (nas quais se desenvolvem as mais diversas atividades, desde a divulgação de informação ao potencial público à prestação de serviços de orientação, de acompanhamento psicológico e/ou pedagógico e de apoio à inserção no mercado de trabalho) e a monitorização institucional das questões ligadas à promoção do sucesso académico parecem favorecer o sucesso dos estudantes que estas instituições acolhem (e.g., Ishler \& Upcraft, 2005; Veloso et al., 2010). Lobo et al. (2011) argumentam que as representações (positivas) dos estudantes sobre a qualidade do ensino ministrado (e.g., práticas pedagógicas, apoio à investigação científica, preparação para o mercado de trabalho regional, nacional ou internacional, etc.), os recursos estruturais e funcionais da instituição (espaços, 
equipamentos, recursos e serviços), a imagem e o prestígio da instituição (tendo por base as atividades de investigação científica que desenvolve, a integração de alunos em projetos nacionais/internacionais que promove ou a sua capacidade de orientar a formação para as necessidades do mercado de trabalho) e o seu envolvimento social e relacional com os atores da instituição afetam (construtivamente) a qualidade de vida dos estudantes, a sua adaptação e realização académica. As interações positivas e frequentes entre o estudante e os atores institucionais (docentes, colegas, funcionários, etc.) e a participação daquele nas diversas atividades promovidas pela instituição (sejam atividades extracurriculares ou resultantes das suas estruturas de apoio) tendem a beneficiar a adaptação ao ensino superior do primeiranista (e.g., Almeida, Guisande, \& Paisana, 2012; Astin, 1997; Cruce, Wolniak, Seifert, \& Pascarella, 2006; Pascarella \& Terenzini, 1991, 2005; Veloso et al., 2010).

As diferentes definições e leituras das causas ou fatores associados ao fenómeno do sucesso académico expõem a sua complexidade e permitem prever a difícil tarefa de desenhar intervenções, em prol da eficácia das mesmas, que incluam um número tão grande (e de natureza tão diversa) de variáveis. Uma intervenção precoce, que apoie os alunos desde o seu ingresso (idealmente, antes deste ocorrer) nos estabelecimentos de ensino terciário, tem a garantia de aumentar as probabilidades de sucesso destes jovens na sua transição para e pelo ensino superior.

\section{PROCESSO DE TRANSIÇÃO E ADAPTAÇÃO AO ENSINO SUPERIOR: CARACTERÍSTICAS E PERSPETIVAS}

Os períodos de transição representam sempre, qualquer que seja o momento do ciclo vital dos indivíduos em que ocorrem, (a perceção de) desequilíbrio (Nico, 2001) ou descontinuidade e são seguidos por um período de adaptação ou ajustamento, que requer, na maior parte dos casos, mudanças nos padrões de resposta comportamental, cognitiva e afetiva (Almeida, Soares, \& Ferreira, 2000; Reich, Harber, \& Siegel, 2008) e a mobilização dos recursos disponíveis (Pinheiro, 2004; Seco, Casimiro, Pereira, Dias, \& Custódio, 2005). A mudança (questão situacional) é, portanto, conceptualmente distinta da transição (questão psicológica) ${ }^{8}$ - a transição consiste, não nas mudanças, mas na perceção e na expressão do indivíduo face às mesmas (Pinheiro, 2004) - e o nível de adaptação (ou o nível de resposta à transição) atingido não pode ser externa e unicamente determinado pelo contexto,

8 A distinção conceptual apresentada é inspirada na abordagem psicológica da transição de Schlossberg (Schlossberg, 1981, 1989; revista e aprofundada por Schlossberg et al., 1995). 
mas interna e necessariamente avaliado pelo indivíduo que vivencia a transição (Schlossberg, Watters, \& Goodman, 1995).

A transição para o ensino superior afigura-se uma das transições mais ambicionadas e simultaneamente mais difíceis que o aluno vivencia (Nico, 1997; Pinheiro, 2004), pelas mudanças de natureza educativa (resultantes do início do ensino terciário), ecológica (decorrente de novos contextos de vida) e desenvolvimental (relacionadas com a realização de tarefas de desenvolvimento esperadas para a fase de vida de um estudante tradicional ${ }^{9}$ : a adolescência tardia ou a adultez emergente) que ocorrem na vida do primeiranista (Pinheiro, 2004; Pittman \& Richmond, 2008). Assim, a par das mudanças desenvolvimentais operadas no final da adolescência e no início da idade adulta, o ingresso no ensino terciário pode ainda confrontar os jovens com múltiplos desafios: sair de casa e separar-se da família e dos amigos, pela primeira vez; enfrentar um meio (totalmente, para a maioria) desconhecido; estabelecer relações com os novos pares e, eventualmente, (para muitos, a primeira relação) de carácter mais íntimo e duradouro; assumir diversas responsabilidades (ligadas à gestão de um orçamento limitado e do tempo para as várias tarefas académicas e não académicas) e tornar-se autónomo; desenvolver a identidade e um sentido para a vida; confrontar-se com um ambiente de ensino-aprendizagem menos estruturado (no qual as normas, expectativas e exigências são mais ténues e ambíguas), ainda que reivindicativo de níveis mais elevados de iniciativa, independência e autonomia na aprendizagem, na gestão de recursos, no estabelecimento de objetivos e na definição das estratégias para os alcançar (Almeida, Ferreira, \& Soares, 1999; Astin, 1993; Chickering \& Reisser, 1993; Diniz, 2005; Figueiredo et al., 2011; Neves \& Pinheiro, 2009; Pascarella \& Terenzini, 2005; Seco et al., 2005; Soares, Almeida, Diniz et al., 2006; Rosário, Nunes, et al., 2010; Rosário, Núñez, \& González-Pienda, 2006).

Apesar de a maioria dos alunos conseguir lidar com a diversidade de mudanças e desafios percecionados e ser bem-sucedida na adaptação, outros experienciam estados emocionais desadaptativos, os quais podem precipitar a retenção, o abandono ou a interrupção dos estudos, durante (particularmente com o termo do primeiro semestre) ou no final do ano letivo (DeBerard et al., 2008; Hillman 2005; Santos, 2007). Alguns estudos apontam para o período que engloba os primeiros dois meses de frequência do ensino superior, como um dos mais críticos do processo de transição e adaptação (Diniz, 2001; Nico, 2001; Pinheiro, 2003, 2004). De acordo com outros autores, a maioria dos alunos que abandona a universidade, fá-lo no final do primeiro semestre ou do primeiro ano letivo (Ishler \& Upcraft, 2005; Sand, Kurpius, \& Rayle, citados em Rayle \& Chung, 2008; Santos, 2007).

9 Entendido como jovem-adulto e estudante a tempo inteiro, que ingressou no ensino superior depois de concluir o ensino secundário, podendo estar deslocado da residência familiar e dependente financeiramente dos pais (Bonnetaud, 2011). 
Tradicionalmente, a análise psicológica deste processo (potencialmente difícil e desafiante) de transição e adaptação ao ensino superior, bem como as intervenções junto dos estudantes (com vista a facilitar este processo e, consequentemente, a promover o sucesso académico), fundamenta-se em conceções de pendor mais internalista ("ancoradas na influência de aspetos psicodinâmicos e desenvolvimentais, etariamente enquadrados, sobre a atualidade experiencial dos estudantes") e de pendor mais externalista ("ancoradas na influência multifacetada do contexto de vida universitário sobre os estudantes”) (Diniz \& Almeida, 2006, p. 30). Dentre estas conceções, há algumas que são sistematicamente citadas na literatura da especialidade, constituindo pontos teóricos de referência na investigação nacional (cf., e.g., Almeida et al., 2012; Diniz \& Almeida, 2006; Jardim, 2007; Pinheiro, 1995, 2003, 2004; Seco et al., 2005; Soares, Almeida, Diniz et al., 2006; Soares, Almeida, \& Ferreira, 2006) e internacional (cf., e.g., Clark, 2005; Inkelas, Daver, Vogt, \& Leonard, 2007; Ishler \& Upcraft, 2005; Luo et al., 2007; Rayle \& Chung, 2008): a articulação entre a abordagem psicológica da transição de Schlossberg (cf. Schlossberg et al., 1995) e a teoria dos sete vetores do desenvolvimento psicossocial dos universitários de Chickering (cf. Chickering \& Reisser, 1993), a teoria do envolvimento institucional de Astin (cf. Astin, 1997), o modelo de integração (académica e social) de Tinto (cf. Tinto, 1993) e o modelo institucional de Pascarella (cf. Pascarella \& Terenzini, 1991, 2005). Se Schlossberg e Chickering enfatizam o protagonismo de cada estudante na sua transição ${ }^{10}$ e no seu desenvolvimento e a importância dos recursos pessoais internos (características individuais, suporte e estratégias) e externos (situação) na capacidade individual de adaptação, os restantes autores destacam o contributo dos estímulos institucionais (clima social/cultural, estrutura/organização, política educativa, entre outras variáveis ligadas à instituição) e sociais (e.g., atitudes/comportamentos e características sociais/culturais das pessoas que ocupam os ambientes institucionais) com os quais o indivíduo interage no seu processo de adaptação ao ensino superior (Diniz \& Almeida, 2006; Ishler \& Upcraft, 2005; Luo et al., 2007; Pinheiro, 2004).

Apesar das diferenças que as separam, todas sublinham a importância de as instituições de ensino serem simultaneamente desafiadoras e apoiantes do primeiranista, focando a sua atenção nas características e experiências do aluno prévias ao ingresso no ensino superior e no ambiente universitário, que se pretende promotor de (de)envolvimento/integração/adaptação, precursores do sucesso académico (na sua definição mais ampla) do estudante. As instituições de ensino terciário, em resposta a este apelo, têm desenvolvido estruturas de apoio de natureza muito diversificada.

10 Entendida como um conceito psicológico, i.e., a transição consiste, não nas mudanças, mas na perceção e na expressão do indivíduo face às mesmas (Pinheiro, 2004). 


\section{ESTRUTURAS DE APOIO AO ALUNO DO ENSINO SUPERIOR PORTUGUÊS: O SUCESSO COMO META}

De um ponto de vista institucional, é importante garantir processos de transição e adaptação ao ensino superior bem-sucedidos, de modo a evitar os desperdícios de recursos institucionais, num período de recursos limitados, e o prejuízo da reputação institucional, desencadeados pelas elevadas taxas de insucesso académico e abandono escolar (Hillman, 2005).

À semelhança das suas congéneres internacionais (Hillman, 2005), e salvaguardando as diferenças resultantes da longa tradição das estruturas de apoio em algumas instituições, sobretudo americanas e inglesas (Pinheiro, 2003), as instituições portuguesas de ensino terciário têm procurado desenvolver estruturas de apoio, entendidas como "todos os mecanismos de divulgação, informação, atração, suporte, integração, orientação e incentivo, cuja meta primordial é a promoção do sucesso" académico do estudante (Veloso et al., 2010, p. 93). A publicação do Despacho n. ${ }^{\circ}$ 6659/99 de 5 de Abril, no qual se solicita a realização de estudos que permitam tipificar as causas de insucesso no ensino superior, de modo a possibilitar o desenvolvimento de medidas remediativas e/ou promotoras de sucesso académico, é apontada por alguns autores (e.g., Brites et al., 2010; Jardim, 2007; Pereira et al., 2006; Santos \& Almeida, 2001) como a expressão legislativa das preocupações institucionais sobre a temática do sucesso académico e o impulso fundamental para a criação mais expressiva de uma das principais estruturas de apoio destas instituições de ensino - as unidades de apoio psicológico e/ou psicopedagógico, podendo também ser genericamente apelidadas de centros de apoio (psicológico e psicoterapêutico, psicopedagógico, de orientação vocacional e profissional, de formação e orientação educativas) ao aluno - e para a proliferação de análises daquela problemática e de estratégias de resposta ${ }^{11}$.

As instituições de ensino tendem a organizar as suas estruturas de apoio em torno dos principais processos/períodos significativos da vida de um estudante deste nível de ensino (Gardner, Upcraft, \& Barefoot, 2005): (1) a tomada de decisão por um curso/instituição e a transição para o ensino superior, (2) a permanência na instituição e, por fim, (3) a transição para o mercado de trabalho (Veloso et

11 Dentre os diversos projetos desenvolvidos em instituições nacionais de ensino superior (em parceria ou isoladamente), é pertinente referir, a título de exemplo, os seguintes: "Estratégias de Promoção do Sucesso Académico no Ensino Superior" (EPSAES-SPASHE; Tavares, 2003; Tavares et al., 2006), "Linha da Universidade de Aveiro" (LUA) (Castanheira, Nogueira, Oliveira, Vasconcelos, \& Pereira, 2010; Pereira, 2005), "Viagem ao Futuro: Programa de Desenvolvimento Vocacional de Apoio à Transição Ensino Secundário-Superior" (Leitão, Paixão, Silva, \& Miguel, 2000), "Estilos de Vida Saudável vs. Insucesso Escolar: Sua Etiologia, Programas de Acção e Estratégias Promotoras de Qualidade" (Pereira et al., 2006), "Transição, Adaptação e Sucesso Académico de Jovens no Ensino Superior" (Almeida et al., 2004), "Cartas do Gervásio ao Seu Umbigo" (Rosário et al., 2006; Rosário, Nunes, et al., 2010), "Formação, Desenvolvimento Pessoal e Sucesso Académico na Educação Superior" (Dias, 2006), "Plano de Apoio à Transição do Ensino Secundário Para o Ensino Superior - Melhor Adaptação; Mais Resiliência; Mais Sucesso” (PAT) (Canavarro, 2009). 
al., 2010). No âmbito desta revisão, interessa sobretudo referir os mecanismos de apoio centrados na primeira fase da vida do primeiranista.

Assim, de acordo com a análise de Veloso et al. (2010) sobre a realidade portuguesa, os mecanismos de comunicação e atração de novos estudantes (que servem propósitos de divulgação e de sedução de candidatos mais informados das opções que tomam, podendo também diminuir o impacto da realidade aquando do ingresso efetivo) compreendem:

- Portais virtuais para potenciais candidatos, através dos quais os interessados obtêm facilmente informação sobre condições de ingresso, expectativas em relação aos candidatos, estruturas e serviços institucionais, oferta formativa, etc.; - Iniciativas (normalmente designadas "Semanas Abertas" e "Cursos/Programas de Verão") de (pré)socialização com o ambiente institucional, que servem também para despertar e/ou afinar vocações e escolhas vocacionais;

- Mostras científicas, feiras vocacionais e/ou de emprego, organizadas para promover a clarividência vocacional e a capacidade de preparação de um projeto académico, sem descurar a vertente publicitária;

- Inquéritos de prospeção centrados no ensino secundário, que permitem conhecer as áreas científicas preferenciais dos seus potenciais candidatos, bem como alguns pormenores do processo de seleção vocacional.

Segundo os mesmos autores, o conjunto de práticas de apoio que as instituições tendem a desenvolver para facilitar o período de transição/adaptação dos novos alunos (ou mecanismos de integração) pode incluir:

- Comissões (frequentemente mistas, integrando elementos da direção do curso e da comissão de praxe do curso) de acolhimento dos alunos recém-chegados, cujos objetivos são assinalar o início de um novo ano letivo, dar as boas-vindas aos diferentes atores institucionais (especialmente aos estreantes na instituição), apresentar o curso e as estruturas que lhe estão afetas e iniciar o processo de socialização estudantil;

- Guia do estudante, quando devidamente estruturado (e atualizado) e difundido (em suporte papel ou através do sistema institucional de informação e comunicação - endereço eletrónico e/ou website dos cursos) constitui um elemento integrador e orientador da atuação dos estudantes;

- Iniciativas inspiradas nos first-year seminars (seminários com mais de um século de existência no sistema educativo superior americano, constituindo um dos preditores mais poderosos da perseverança dos primeiranistas; Ishler \& Upcraft, 2005), como ciclos de sessões (in)formativas que podem variar na sua estrutura e organização 
(dependendo da configuração assumida - conferência, workshop, programa de intervenção, curso breve ou disciplina de opção livre ${ }^{12}$ - assim apresentam diferentes durações e frequências das sessões, conteúdos, métodos pedagógicos, expectativas de resultado, etc.) e nas temáticas que abordam (e.g., estratégias de aprendizagem, gestão do tempo, competências sociais, comunicação, utilização de infraestruturas bibliográficas, produção de trabalhos científicos), mas que estão unidas pelo objetivo comum de dotar os alunos de conhecimentos, estratégias e competências que lhes permitam fazer face às novas exigências académicas, sociais e desenvolvimentais; - Unidades de apoio ao estudante com necessidades educativas especiais, responsáveis pela interface direta com este tipo de estudantes e pelo apoio, sobretudo, ao processo de ensino-aprendizagem (e.g., disponibilizando documentos traduzidos para braille, gravando aulas em suporte audiovisual, desenvolvendo um regulamento para a avaliação de estudantes com este tipo de necessidades);

- Programas de ajuda sustentados em modelos de apoio de pares (sistema de ajuda a alunos por alunos, destinado a atuar, complementarmente, nos problemas de natureza emocional ou outros, peer counselling/support, na integração social, peer mentoring, ou nas dificuldades académicas, peer tutoring; Pereira, 2005), de mentorado (sistema de ajuda a estudantes por mentores, i.e., indivíduos mais experientes, normalmente colegas mais experientes ou docentes, que servem de guias e modelos na adaptação ao novo ambiente) e de tutorado (sistema de ajuda a alunos por tutores, indivíduos mais experientes, geralmente estudantes mais experientes ou docentes, que proporcionam sobretudo orientação académica formal), dos quais resultam vantagens para todos os participantes (apoiantes e apoiados) e para a instituição (quando assentes num sistema de voluntariado, estes programas comportam baixos custos) ${ }^{13}$;

- Inquéritos (normalmente associados ao ato da matrícula do novo aluno), quando devidamente aplicados, analisados e difundidos os seus resultados, podem facultar informação favorecedora de um planeamento do $1^{\circ}$ ano curricular melhor (que atenue os desfasamentos associados aos níveis de ensino secundário-superior e ao início de um novo ciclo de estudos).

12 Ainda que as configurações de conferência e workshop sejam as mais praticadas (provavelmente devido às menores exigências de planeamento/aplicação/avaliação), a última década tem sido profícua na organização e concretização de programas de intervenção [e.g., "Cartas do Gervásio ao Seu Umbigo" (Rosário et al., 2006), "Programa de Desenvolvimento de Competências Intrapessoais, Interpessoais e Profissionais" (PDCIIP; Jardim, 2007), "Stress em Linha” (Oliveira, 2008)], de cursos breves de desenvolvimento de competências de leitura e de expressão oral/escrita na Língua Portuguesa e Inglesa ou de consolidação de conhecimentos basilares técnico-científicos de Matemática, Física ou Química (e.g., oferta formativa no âmbito das Linha 1 e 3 do projecto PAT; Canavarro, 2009) ou de disciplinas de opção livre (e.g., disciplina E.P.S.A.E.S; Tavares et al., 2006)].

13 Em Portugal, começam a proliferar alguns programas desenvolvidos com base nestes modelos de apoio: "Apoio a Alunos por Alunos - Peer Counselling/Support" (Pereira et al., 2005), nas Universidades de Aveiro e Coimbra; "Elos de Apoio" (Carreiro, 2005), na Universidade de Évora; "Programa de Adaptação à Faculdade” (PAF; Fernandes, 2005), na Faculdade de Ciências da Universidade de Lisboa; "Programa de Monitorização e Tutorado" (Gonçalves, 2010), no Instituto Superior Técnico da Universidade Técnica de Lisboa; entre outros. 
Veloso et al. (2010), no seu trabalho de identificação de estruturas de apoio das instituições portuguesas, apontam ainda os mecanismos de acompanhamento e/ou orientação, "situados a jusante do ensino superior (...) procuram prestar o acompanhamento e/ou orientação de que os alunos necessitam no decurso do seu percurso (...) contribuir para a melhoria contínua e para uma melhor adequação entre ensino e aprendizagem" (pp. 105-106), e os mecanismos de inserção profissional, que podem apoiar não só os estudantes e os diplomados no processo de transição para o mercado de trabalho (através de diversas atividades: aconselhamento e/ou orientação profissional, intervenções promotoras de competências úteis na procura e candidatura a oportunidades de emprego, divulgação de oportunidades de emprego e formação, etc.), como também às "demais unidades orgânicas no interface desejável com a estrutura económico-produtiva que envolve a instituição" (p. 117). Para exemplificar estes mecanismos enunciam as unidades de aconselhamento psicológico e/ou psicopedagógico, os observatórios pedagógicos (que se ocupam da avaliação pedagógica e do desenvolvimento de iniciativas que visem a deteção e eliminação de dificuldades no processo ensino-aprendizagem), os provedores do estudante (docentes cuja ação se desenvolve em articulação com as associações de estudantes e com os órgãos e serviços da instituição de ensino), a carta de direitos e deveres dos diferentes atores institucionais, as bolsas de estudo e os prémios pecuniários de caráter meritório, bem como as unidades de inserção na vida ativa (UNIVA), os protocolos e as parcerias institucionais, as bolsas de emprego (infraestrutura de base digital que permite a interação entre candidatos e empregadores) e os observatórios de emprego (atentos à monitorização da inserção profissional dos alunos recém-licenciados).

Alguns dos mecanismos de apoio - como as unidades de aconselhamento psicológico e/ou psicopedagógico, as unidades de apoio ao estudante com necessidades educativas especiais, os programas de apoio (de pares, de mentorado ou de tutorado), o guia do estudante, entre outros - podem ter uma atuação transversal a todos os períodos significativos da vida de um estudante do ensino superior, visando a sua tomada de decisão informada, a sua integração na nova instituição de ensino, o acompanhamento e orientação na sua estada no ensino superior e a sua inserção no mercado de trabalho (Veloso et al., 2010).

Por último, importa ainda referir que, apesar de o desenvolvimento das estruturas de apoio das instituições portuguesas de ensino superior ter ainda um longo caminho a percorrer, particularmente no que diz respeito à produção de regulamentação própria (da qual depende o apoio institucional a nível económico, logístico e de recursos humanos; Pinheiro, 2003) que auxilie e expanda as diversas atividades dos centros de apoio aos estudantes, é notável o esforço de todos os que colaboram (técnicos, docentes, investigadores, voluntários) nestas atividades de facilitação do sucesso pessoal, social e académico do aluno e procuram otimizar esta prática. A existência da 
Rede dos Serviços de Apoio Psicológico do Ensino Superior - Associação Profissional (RESAPES - AP), desde 2004, é o lado mais visível deste esforço de homogeneização, otimização e fundamentação da prática dos centros de apoio ao estudante, "ao nível da ética e etiologia de base, do reconhecimento institucional dos serviços e da colaboração interinstitucional na partilha de informação e definição de boas práticas” (Pereira Castanheira, Melo, Ferreira, \& Vagos, 2010, p. 5).

\section{CONCLUSÃO}

O conhecimento veiculado pela educação tem um papel fulcral no desenvolvimento humano e socioeconómico de qualquer democracia, pelo que o acesso e a permanência de todos os cidadãos no ensino formal, em particular, no ensino superior, devem ser garantidos pelas sociedades. Quando comparado com outros países da União Europeia, Portugal mantém alguns indicadores desfavoráveis nesta matéria, apesar das importantes transformações introduzidas no sistema de ensino terciário desde os últimos anos do Estado Novo e do seu esforço mais recente na construção de um espaço europeu coerente e compatível de ensino superior. A investigação direcionada para a compreensão (e manipulação) do sucesso académico, bem como do processo de transição e adaptação ao ensino superior, constituiu uma das estratégias válidas para a retificação desses indicadores.

Neste artigo apresentaram-se (de forma não exaustiva) definições, perspetivas e dados provenientes da investigação sobre os dois fenómenos anteriores e as respostas das instituições nacionais de ensino superior com o objetivo de os promover, para concluir o seguinte. Por um lado, só definições amplas de sucesso académico (e a utilização de medidas que contemplam os seus aspetos escolares, sociorrelacionais e psicológicos) e explicações integradoras da constelação de fatores implicados são válidas - facto que complexifica o desenho, a implementação e a avaliação de intervenções. Por outro, a transição para o ensino superior afigura-se difícil (pelas mudanças de natureza educativa, ecológica e desenvolvimental que implica) e boa parte do sucesso das fases posteriores (permanência na instituição de ensino e saída da instituição/ progressão para o mercado de trabalho) é determinada pelo desfecho bem-sucedido desta fase inicial. Por fim, e no seguimento do argumento anterior, é necessário que as instituições portuguesas de ensino terciário mantenham e aprimorem as diversas estruturas de apoio (particularmente ao estudante recém-chegado), de modo a evitar desperdícios de recursos institucionais (num período de recursos limitados) e o prejuízo da reputação institucional, ao mesmo tempo que cumprem o seu importante papel de coprotagonista na construção de uma Europa baseada no conhecimento. 


\section{REFERÊNCIAS}

Alarcão, I. (2000). Para uma conceptualização dos fenómenos de insucesso/sucesso escolares no ensino superior. In J. Tavares \& R. Santiago (Orgs.), Ensino superior: (In)Sucesso académico (pp. 11-23). Porto: Porto Editora.

Almeida, L. (2002). Formatar o ensino a pensar na aprendizagem. In A. Pouzada, L. Almeida, \& R. Vasconcelos (Eds.), Contextos e dinâmicas da vida académica (pp. 239-252). Guimarães: Universidade do Minho.

Almeida, L., Ferreira, J., \& Soares, A. (1999). Questionário de Vivências Académicas: Construção e validação de uma versão reduzida (QVA-r). Revista Portuguesa de Pedagogia, 33(3), 181-207.

Almeida, L., Gonçalves, A., Soares, A., Marques, A., Machado, C., \& Fernandes, E. (2004). Transição, adaptação e rendimento académico de jovens no ensino superior (relatório final de projeto). Braga: Universidade do Minho.

Almeida, L., Guisande, M., \& Paisana, J. (2012). Extra-curricular involvement, academic adjustment and achievement in higher education: A study of Portuguese students. Anales de Psicología, 28(3), 860-865.

Almeida, L., Guisande, M., Soares, A., \& Saavedra, L. (2006). Acesso e sucesso no ensino superior em Portugal: Questões de género, origem socio-cultural e percurso académico dos alunos. Psicologia: Reflexão e Crítica, 19(3), 507-514.

Almeida, L., Soares, A., \& Ferreira, J. (2000). Transição e adaptação à universidade: Apresentação do Questionário de Vivências Académicas (QVA). Psicologia, 14(2), 189-208.

Amante, M. (2007). A avaliação da qualidade no ensino superior: Uma proposta de indicadores de qualidade docente (Tese de doutoramento não publicada). Universidade de Coimbra, Coimbra.

Araújo, B., \& Almeida, L. (2010, Julho). Interferência dos factores psicossociais e curriculares no rendimento académico: Um estudo com estudantes de Enfermagem. Comunicação apresentada no I Seminário Internacional "Contributos da Psicologia em Contextos Educativos", Universidade do Minho, Braga.

Astin, A. (1997). What matters in college? Four critical years revisited. San Francisco: Jossey-Bass.

Bonnetaud, C. (2011). Student engagement profile: A comparison of traditional and nontraditional undergraduate college students. Raleigh, NC State: North Carolina State University.

Brites, J., Seco, G., Canastra, F., Dias, I., \& Abreu, M. (2010). (In)sucesso académico no ensino superior: Factores e estratégias de intervenção. In A. Pereira, H. Castanheira, A. Melo, A. Ferreira, \& P. Vagos (Eds.), Apoio psicológico no ensino superior: Modelos e práticas - Actas do I Congresso Nacional da RESAPES-AP (pp. 152-159). Aveiro: Universidade de Aveiro.

Bruinsma, M., \& Jansen, E. (2007). Educational productivity in higher education: An examination of part of the Walberg educational productivity model. School Effectiveness and School Improvement, 18(1), 45-65.

Canavarro, J. (2007). Para a compreensão do abandono escolar. Lisboa: Texto Editores.

Canavarro, J. (2009). Quinze meses de Plano de Apoio à Transição na UC. Rua Larga, 24. Consultado em http://www.uc.pt/rualarga/anteriores/24/24_03

Carreiro, A. (2005). Projecto Elos de Apoio: Um exemplo de voluntariado universitário. In A. Pereira \& E. Motta (Eds.), Acção social e aconselhamento psicológico no ensino superior: Investigação e intervenção - Actas do Congresso Nacional (pp. 223-236). Coimbra: SASUC Edições.

Castanheira, H., Nogueira, R., Oliveira, P., Vasconcelos, G., \& Pereira, A. (2010). Acção social no ensino superior: Novos contributos para uma agenda estratégica. In A. Pereira, H. Castanheira, A. 
Melo, A. Ferreira, \& P. Vagos (Eds.), Apoio psicológico no ensino superior: Modelos e práticas - Actas do I Congresso Nacional da RESAPES-AP (pp. 96-105). Aveiro: Universidade de Aveiro. Chickering, A., \& Reisser, L. (1993). Education and Identity. San Francisco: Jossey-Bass.

Clark, M. (2005). Negotiating the freshman year: Challenges and strategies among first-year college students. Journal of College Student Development, 46(3), 296-316.

Comissão das Comunidades Europeias. (2003, Fevereiro). Comunicação da Comissão: O papel das universidades na Europa do conhecimento. Consultado em http://eur-lex.europa.eu/legal-content/EN/TXT/?uri=URISERV\%3Ac11067

Crespo, V. (2006). Rede das instituições de ensino superior nos países da união europeia. Revista Lusófona de Educação, 7, 41-57.

Cruce, T., Wolniak, G., Seifert, T., \& Pascarella, E. (2006). Impacts of good practices on cognitive development, learning orientations, and graduate degree plans during the first year of college. Journal of College Student Development, 47(4), 365-383.

Curado, A., \& Machado, J. (2005). Percursos escolares dos estudantes da Universidade de Lisboa: Factores de sucesso e insucesso escolar na Universidade de Lisboa. Lisboa: Universidade de Lisboa.

DeBerard, M., Spielmans, G., \& Julka, D. (2004). Predictors of academic achievement and retention among college. College Student Journal, 38(1), 66-80.

Dias, G. (Coord.). (2006). Apoio psicológico a jovens do ensino superior: Método, técnicas e experiências. Porto: Edições ASA.

Diniz, A. (2001). Crenças, escolha de carreira e integração universitária (Tese de doutoramento não publicada). Universidade do Minho, Braga.

Diniz, A. (2005). A universidade e os seus estudantes: Um enfoque psicológico. Lisboa: Edições ISPA.

Diniz, A., \& Almeida, L. (2006). Adaptação à universidade em estudantes do primeiro ano: Estudo diacrónico da interacção entre o relacionamento com pares, o bem-estar pessoal e o equilíbrio emocional. Análise Psicológica, 24(1), 29-38.

Fernandes, C. (2005). PAF: Como intervir precocemente com estudantes do ensino superior. In A. Pereira \& E. Motta (Eds.), Acção social e aconselhamento psicológico no ensino superior: Investigação e intervenção - Actas do Congresso Nacional (pp. 227-235). Coimbra: SASUC Edições.

Ferreira, J., Almeida, L., \& Soares, A. (2001). Adaptação académica em estudantes do $1^{\circ}$ ano: Diferenças de género, situação de estudante e curso. Psico-USF, 6(1), 1-10.

Figueiredo, C., Maia, J., \& Pinheiro, M. (2004). Percepção da aceitação: Dados preliminares de um estudo de validação com estudantes da Universidade de Coimbra. In C. Machado, L. Almeida, M. Gonçalves, \& V. Ramalho (Orgs.), Actas da X Conferência Internacional Avaliação Psicológica: Formas e Contextos (pp. 180-188). Braga: Psiquilíbrios Edições.

Figueiredo, C., Maia, J., \& Pinheiro, M. (2011). Avaliação da percepção da aceitação da família e dos amigos: Contributos para a validade da estrutura factorial da Perceived Aceptance Scale (PAS). Psychologica, 53, 275-298.

Gardner, J., Upcraft, M., \& Barefoot, B. (2005). Conclusion: Principles of good practice for the first college year and summary of recommendations. In M. Upcraft, J. Gardner, B. Barefoot, \& Associates (Eds.), Challenging and supporting the first-year student: A handbook for improving the first year of college ( $1^{\text {st }}$ ed., pp. 27-12). EUA: John Wiley \& Sons.

Gold, A., \& Souvignier, E. (2005). Prediction of college outcomes: Results from longitudinal studies. Zeitschrift für Entwicklungspsychologie und Pädagogische Psychologie, 37(4), 214-222.

Gonçalves, I. (2010). Contributos dos modelos da auto-regulação da aprendizagem para a formação de alunos e professores no ensino superior. In A. Pereira, H. Castanheira, A. Melo, A. Ferreira, \& 
P. Vagos (Eds.), Apoio psicológico no ensino superior: Modelos e práticas - Actas do I Congresso Nacional da RESAPES-AP (pp. 597-610). Aveiro: Universidade de Aveiro.

Hillman, K. (2005). The first year experience: The transition from secondary school to university and TAFE in Australia. (Research Report No 40, Longitudinal Surveys of Australian Youth). Camberwell, Victoria: Australian Council for Educational Research. Consultado em http:// research.acer.edu.au/cgi/viewcontent.cgi?article=1043\&context=lsay_research

Inkelas, K., Daver, Z., Vogt, K., \& Leonard, B. (2007). Living-learning programs and first-generation college students' academic and social transition to college. Research in Higher Education, 48(4), 403-434.

Ishler, J., \& Upcraft, M. (2005). The keys to first-year student persistence. In M. Upcraft, J. Gardner, B. Barefoot, \& Associates (Eds.), Challenging and supporting the first-year student: A handbook for improving the first year of college ( $1^{\text {st }}$ ed., pp. 27-12). EUA: John Wiley \& Sons.

Jardim, M. (2007). Programa de desenvolvimento de competências pessoais e sociais: Estudo para a promoção do sucesso académico (Tese de doutoramento não publicada). Universidade de Aveiro, Aveiro.

Leitão, L., Paixão, M., Silva, J., \& Miguel, J. (2000). Viagem ao Futuro: Programa de desenvolvimento vocacional de apoio à transição ensino secundário/ensino superior. In A. Soares, A. Osório, J. Capela, L. Almeida, R. Vasconcelos, \& S. Caires (Eds.), Transição para o ensino superior (pp. 215-222). Braga: Universidade do Minho.

Lobo, F., Almeida, L., \& Pinheiro, M. (2011). Avaliação do clima académico no ensino superior. In A. Ferreira, A. Verhaeghe, R. Silva, L. Almeida, R. Lima, \& S. Fraga (Eds.), Actas de VIII Congresso Iberoamericano de Avaliação/Evaluación Psicológica e XV Conferência Internacional Avaliação Psicológica Formas e Contextos (pp. 1364-1375). Lisboa: Sociedade Portuguesa de Psicologia.

Luo, M., Williams, J., \& Vieweg, B. (2007). Transitioning transfer students: Interactive factors that influence first-year retention. College and University, 83(2), 8-19.

Mills, C., Heyworth, J., Rosenwax, L., Carre, S., \& Rosenberg, M. (2009). Factors associated with the academic success of first year Health Science students. Advances in Health Science Education, 14, 205-217.

Neves, C., \& Pinheiro, M. (2009). A qualidade dos relacionamentos interpessoais com os amigos: Adaptação e validação do Quality of Relationships Inventory (QRI) numa amostra de estudantes do ensino superior. EXEDRA, 2, 9-32.

Nico, B. (1997). A adaptação à Universidade: Fragmentos de um percurso curricular. In Actas da Conferência Internacional - A Informação e a Orientação Escolar e Profissional no Ensino Superior: Um desafio da Europa (pp. 105-114). Coimbra: Universidade de Coimbra.

Nico, J. (2001). A adaptação do(a) estudante à universidade: Porque não também o contrário. In R. Sousa, E. Sousa, F. Lemos, \& C. Januário (Orgs.), III Simpósio Pedagogia na Universidade (pp. 55-67). Lisboa: Reitoria da Universidade Técnica de Lisboa.

Nunes, M. (2006). (In)sucesso escolar no ensino superior: Variáveis biopsicossociais. Castelo Branco: Politécnica.

Oliveira, T. (2008). Stress em Linha: Programa de intervenção no ensino superior (Dissertação de mestrado não publicada). Universidade de Aveiro, Aveiro.

Pacheco, A. (2008). Estilos de vida, qualidade de vida pessoal, motivação e sucesso académico: $O$ caso da Universidade do Algarve (Dissertação de mestrado não publicada). Universidade do Algarve, Faro.

Pascarella, E., \& Terenzini, P. (1991). How college affects students: Findings and insights from twenty years of research. San Francisco: Jossey-Bass. 
Pascarella, E., \& Terenzini, P. (2005). How college affects students: A third decade of research (Vol. 2). San Francisco: Jossey-Bass.

Pereira, A. (2005). Para obter sucesso na vida académica: Apoio dos estudantes pares. Aveiro: Universidade.

Pereira, A., Castanheira, H., Melo, A., Ferreira, A., \& Vagos, P. (Eds.). (2010). Nota de abertura. In Apoio psicológico no ensino superior: Modelos e práticas - Actas do I Congresso Nacional da RESAPES-AP (pp. 5-6). Aveiro: Universidade de Aveiro.

Pereira, A., Motta, E., Vaz, A., Pinto, C., Bernardino, O., Melo, A.,... Lopes, P. (2006). Sucesso e desenvolvimento psicológico no ensino superior: Estratégias de intervenção. Análise Psicológica, 24(1), 51-59.

Pereira, A., Motta, E., Vaz, A., Pinto, C., Bernardino, O., Melo, A.,... Queiroz, A. (2005). Preciso de alguém que me oiça: Intervenção de peer counselling/support nas residências universitárias. In A. Pereira \& E. Motta (Eds.), Acção social e aconselhamento psicológico no ensino superior: Investigação e intervenção - Actas do Congresso Nacional (pp. 187-194). Coimbra: SASUC Edições.

Pinheiro, M. (1995). O domínio das emoções e o desenvolvimento da autonomia: Contributos para o estudo do desenvolvimento psicossocial do estudante universitário (Dissertação de mestrado não publicada). Universidade de Coimbra, Coimbra.

Pinheiro, M. (2003). Uma época especial: Suporte social e vivências académicas na transição e adaptação ao ensino superior (Tese de doutoramento não publicada). Universidade de Coimbra, Coimbra.

Pinheiro, M. (2004). O desenvolvimento da transição para o ensino superior: O princípio depois de um fim. Aprender, 29, 9-20.

Pinheiro, M. (2010). Princípios e desafios para boas práticas dos estudantes no ensino superior: Uma proposta de operacionalização. In A. Pereira, H. Castanheira, A. Melo, A. Ferreira, \& P. Vagos (Eds.), Apoio psicológico no ensino superior: Modelos e práticas - Actas do I Congresso Nacional da RESAPES-AP (pp. 219-232). Aveiro: Universidade de Aveiro.

Pinheiro, M., \& Ferreira, J. (2002). O Questionário de Suporte Social: Adaptação e validação da versão portuguesa do Social Support Questionnaire (SSQ6). Psychologica, 30, 315-333.

Pinheiro, M., \& Ferreira, J. (2006). A Escala de Provisões Sociais: Uma medida da percepção do suporte social. Psicologia e Educação, 5(1), 49-62.

Pittman, L., \& Richmond, A. (2008). University belonging, friendship quality, and psychological adjustment during the transition to college. The Journal of Experimental Education, 76(4), 343-361.

Ponte, J. (2005). O processo de Bolonha e a formação inicial de professores em Portugal. In J. Serralheiro (Org.), O processo de Bolonha e a formação dos educadores e professores portugueses (pp. 63-73). Porto: Profedições.

Rayle, A., \& Chung, K. (2008). Revisiting first-year college student's mattering: Social support, academic stress, and the mattering experience. Journal of College Student Retention, 9(1), 21-37.

Rego, C., \& Caleiro, A. (2010). O "Mercado" do Ensino Superior em Portugal: Um diagnóstico da situação actual (Documento de Trabalho no 2010/04). Consultado em http://dspace.uevora.pt/rdpc/ bitstream/10174/8474/1/wp_2010_04.pdf

Reich, W., Harber, K., \& Siegel, H. (2008). Self-structure and well-being in life transitions. Self and Identity, 7, 129-150.

Rosário, P., Nunes, T., Magalhães, C., Rodrigues, A., Pinto, R., \& Ferreira, P. (2010). Processos de auto-regulação da aprendizagem em alunos com insucesso no $1 .^{\circ}$ ano de Universidade. Revista Semestral da Associação Brasileira de Psicologia Escolar e Educacional, 14(2), 349-358.

Rosário, P., Núñez, J., \& González-Pienda, J. (2006). Cartas do Gervásio ao seu umbigo: Comprometer-se com o estudar na universidade. Coimbra: Edições Almedina, S.A.. 
Rosário, P., Núñez, J., González-Pienda, J., Valle, A., Trigo, L., \& Guimarães, C. (2010). Enhancing self-regulation and approaches in first-year college students: A narrative-based program assessed in the Iberian Peninsula. European Journal of Psychology of Education, 25, 411-428.

Ruthig, J., Marrone, S., Hladkyj, S., \& Robinson-Epp, N. (2011). Changes in college student health: Implications for academic performance. Journal of College Student Development, 52(3), 307-320.

Santos, J., \& Pinheiro, M. (2010). Assiduidade às aulas, satisfação com o curso e estratégias de motivação para a aprendizagem em estudantes do ensino superior. In A. Pereira, H. Castanheira, A. Melo, A. Ferreira, \& P. Vagos (Eds.), Apoio psicológico no ensino superior: Modelos e práticas Actas do I Congresso Nacional da RESAPES-AP (pp. 362-370). Aveiro: Universidade de Aveiro.

Santos, L., \& Almeida, L. (2001). Vivências académicas e rendimento escolar: Estudo com alunos universitários do $1 .^{\circ}$ ano. Análise Psicológica, 19(2), 205-217.

Santos, P. (2007). A relação das vivências e expectativas académicas com o abandono escolar no ensino superior (Dissertação de mestrado não publicada). Universidade de Coimbra, Coimbra.

Schlossberg, N. (1981). A model for analysing human adaptation to transition. The Counseling Psychologist, 9(2), 2-18.

Schlossberg, N. (1989). Overwhelmed: Coping with life's ups and downs. Lexington, MA: Lexington Books.

Schlossberg, N., Watters, E., \& Goodman, J. (1995). Counselling adults in transition: Linking practice with theory. NY: Springer Publishing Company.

Seco, G., Casimiro, M., Pereira, M., Dias, M., \& Custódio, S. (2005). Para uma abordagem psicológica da transição do ensino secundário para o ensino superior: Pontes e alçapões. Leiria: Instituto Politécnico de Leiria.

Silva, C., \& Ribeiro, I. (2007). Auto-regulação: Influência no sucesso escolar dos alunos universitários. Psicologia: Teoria, Investigação e Prática, 1, 139-150.

Soares, A., Almeida, L., Diniz, A., \& Guisande, M. (2006). Modelo Multidimensional de Ajustamento de jovens ao contexto Universitário (MMAU): Estudo com estudantes de ciências e tecnologias versus ciências sociais e humanas. Análise Psicológica, 24(1), 15-27.

Soares, A., Almeida, L., \& Ferreira, J. (2006). Questionário de Vivências Académicas: Versão integral (QVA) e versão reduzida (QVA-r). In M. Gonçalves, L. Almeida, \& M. Simões (Coords.), Avaliação psicológica: Instrumentos validados para a população portuguesa (2a ed. atualizada, Vol. 1, pp.101-120). Coimbra: Quarteto.

Tavares, J. (2003). Formação e inovação no ensino superior. Porto: Porto Editora.

Tavares, J., \& Huet, I. (2001). Sucesso académico no ensino superior: Um olhar sobre o professor universitário. In R. Sousa, E. Sousa, F. Lemos, \& C. Januário, III Simpósio Pedagogia na Universidade (pp. 149-160). Lisboa: Reitoria da Universidade Técnica de Lisboa.

Tavares, J., Pereira, A., Gomes, A., Cabral, A., Fernandes, C., Huet, I.,... Monteiro, S. (2006). Estratégias de promoção do sucesso académico: Uma intervenção em contexto curricular. Análise Psicológica, 24(1), 61-72.

Tavares, J., \& Silva, I. (2001). Sucesso académico no ensino superior. In R. Sousa, E. Sousa, F. Lemos, \& C. Januário (Orgs.), III Simpósio Pedagogia na Universidade (pp. 55-67). Lisboa: Reitoria da Universidade Técnica de Lisboa.

Taveira, M. (2000). Sucesso no ensino superior: Uma questão de adaptação e de desenvolvimento vocacional. In J. Tavares \& R. Santiago (Orgs.), Ensino superior: (In)Sucesso académico (pp. 51-71). Porto: Porto Editora.

The Organisation for Economic Co-operation and Development (OECD). (2015). Regards sur l'éducation 2015: Les indicateurs de l'OCDE. Paris: OECD Publishing. 
Tinto, V. (1993). Leaving college: Rethinking the causes and cures of student attrition ( $\left.2^{\text {nd }} \mathrm{ed}.\right)$. Chicago: University of Chicago Press.

Upcraft, M., Gardner, J., \& Barefoot, B. (2005). Introduction: The first year of college revisited. In M. Upcraft, J. Gardner, B. Barefoot, \& Associates (Eds.), Challenging and supporting the first-year student: A handbook for improving the first year of college ( $1^{\text {st }}$ ed., pp. 1-12). EUA: John Wiley \& Sons.

Veloso, H., Costa, A., \& Lopes, J. (Coords.). (2010). Factores, representações e práticas institucionais de promoção do sucesso escolar no ensino superior. Porto: U. Porto editorial.

Zajacova, A., Lynche, S., \& Espenshade, T. (2005). Self-efficacy, stress, and academic success in college. Research in Higher Education, 46(6), 677-706.

Zimmerman, B. (2008). Investigating self-regulation and motivation: Historical background, methodological developments, and future prospects. American Educational Research Journal, 45, 166-183. 